International Journal on Soft Computing ( IJSC ), Vol.2, No.1, February 2011

\title{
EVOLVING RULES USING GENETIC FUZZY APProACH - AN EduCATIONAL CASE STUdY
}

\author{
Kunjal Mankad ${ }^{1}$, Priti Srinivas Sajja ${ }^{2}$ and Rajendra Akerkar ${ }^{3}$ \\ 1Assistant Professor, ISTAR, CVM, Vallabh Vidyanagar, India, kunjal_mankad@yahoo.com \\ 2 Associate Professor, Sardar Patel University, India, priti_sajja@yahoo.com \\ 3 Senior Researcher, Vestlandsforsking, Norway, akerkar8@gmail.com
}

\begin{abstract}
The work presents design and development of a system to automatically evolve rules through genetic-fuzzy approach. The work highlights the advantages of genetic and fuzzy hybridization and proposes a framework to evolve rules automatically. The objective of the framework is to reduce the development effort and guide the development procedure in user friendly fashion. The architecture presents a novel approach which integrates fuzzy logic with system design as well as back end of the framework is used to evolve the fuzzy rules using genetic algorithm approach. To experiment the working of the proposed framework, a system to measure multiple intelligence is selected. The paper describes the detail methodology including encoding strategy of rules, suitable genetic operators and fitness function to evolve fuzzy rules for the selected system. The initial population of rules, evolved generations and output results are also described. The paper concludes with the scope and applications of the work to other domains.
\end{abstract}

\section{KEY WORDS}

Information and Communication Technology (ICT), Genetic Algorithms, Fuzzy Logic, Genetic Fuzzy Systems, Rule Base, Theory of Multiple Intelligence

\section{INTRODUCTION}

Soft computing techniques are more powerful and efficient as they provide feasible and less costly solutions compared to hard computing techniques. There are several methods of soft computing family from which Fuzzy Logic (FL) and Genetic Algorithm (GA) are the most important techniques. With fuzzy logic based technique, imprecision, uncertainty and human oriented knowledge representation is possible; still self learning and generalization of rules can not be possible. Hence, the need is arise for hybridization of FL approach with Genetic Algorithm. Clever combination of Genetic Algorithms and Fuzzy Logic offers advantages of both the fields. The paper focuses on evolving rule based model for identification of multiple intelligence in human beings by utilizing Genetic-Fuzzy hybrid approach.

ICT has introduced numerous trends in different areas of human life with integration of education field. As a result; more employment and increased demand for multiple skills are generated. Many times; it has been observed that an individual himself can not identify his own interest and capabilities in specific areas due to improper educational methods. The solution of this problem is to identify the capabilities of an individual since childhood. Out of the different theories available to identify and enhance human intelligence, theory of Multiple Intelligence (MI) has been pioneer among researchers and educationalists.

The proposed application considers a novel approach of automatic evolution of rules for identifying multiple intelligence. As a part of research work, architecture of evolving system is designed to satisfy the need of decision support using genetic fuzzy approach. In this approach, 
the initial population of knowledge base requires a few encoded rules, suggested by administrator of the system to initiate the process of evolution. The system in its evolutionary period evolves new rules within the knowledge base through specially designed operators along with the fitness function designed for the domain. The remaining chapter is organized as follows. Section 2 discusses background of MI theory and various types of intelligence and work done so far in the area. Section 3 establishes need of genetic fuzzy approach for the domain along with brief literature survey. Section 4 presents architecture of the proposed system with detailed methodology. Section 5 discusses experimental results of the system and section 6 concludes the paper with future scope.

\section{Educational Perspective}

In today's competitive world, it is very important to select appropriate career in order to achieve success by utilizing ones' capabilities and intelligence. The originator of the theory of multiple intelligences, Howard Gardner, defines intelligence as potential ability to process a certain sort of information [1]. However, there is also a possibility of many other types of intelligence in individuals [2]. All types of intelligence play an important role in overall growth of human capabilities. It has been proven that specific types of intelligence such as logical, verbal, interpersonal, kinesthetic etc. are essential to have satisfactory level of success in the field of science and technology, management, sports, etc. [3]. Among all, the technical and managerial abilities play a critical role in one's success. The proposed approach highlights classification of users' managerial and technical skills with genetic fuzzy approach. The types of human intelligence according to theory of MI are as follows:

$>$ Logical Intelligence: Ability to deal with numerical aptitude

$>$ Verbal Intelligence: Capacity to learn and understand using language

$>$ Interpersonal Intelligence: Ability to understand other people

$>$ Intrapersonal Intelligence: Ability to understand oneself

$>$ Musical Intelligence: Ability to understand and learn music

$>$ Naturalist Intelligence: Ability to discriminate among living things

$>$ Kinesthetic Intelligence: Capacity of using whole body to solve something

$>$ Moral and Existential Intelligence: Concerns with ultimate issues and changing attitude

The field of education and technology has contributed numerous research projects by implementing Theory of MI for the last few decades, some of them are as follows [4, 5, 6, 7, 8, 9, $10,11,12,13]$ :

International educational online learning programs for students as well as teachers

$>$ Curriculum planning, parents' interaction, etc.

$>$ Research based on school students of different ages with IQ tests to identify their skills

$>$ Adult developmental programs

$>$ Employees' developmental programs

$>$ New AI approach for students' academic performance using fuzzy rule generation

$>$ The research project "EDUCE", implemented as a predictive system using MI

$>$ Application of the Theory of Multiple Intelligences to Digital Systems Teaching

$>$ Learning style improvement using information technology, and many more

\section{GeNETIC FuzzY SySTEMS}

Genetic Algorithms (GA) are robust general purpose search algorithms that use principles inspired by natural population genetics to evolve solutions to the problem. GA provides flexibility to interface with existing models and easy to hybridize [14]. On other hand; Fuzzy Logic (FL) based systems are popular for handling uncertainty and imprecision in real situation easily. But 
the major limitation of such systems is that they are not able to learn [15] as well as require documentation of knowledge which needs further continuous maintenance. Hence, hybridization of FL with GA becomes essential to achieve advantages both the aforementioned approaches. In such systems, knowledge in the form of linguistic variables, fuzzy membership function parameters, fuzzy rules, number of rules, etc. can be converted into suitable candidate solutions through generic code structure of GA. Enlisted examples are very useful real world applications those dealing with intelligent information systems where genetic fuzzy methodology has been successfully implemented.

$>$ Diagnostic system for decease such as myocardial infraction, breast cancer, diabetes, dental development age prediction, abdominal pain, etc. [14, 15, 16, 17]

$>$ A trading system with GA for optimized fuzzy model [18]

$>$ For optimizing social regulation policies [19]

$>$ Self integrating knowledge-based brain tumor diagnostics system [20]

$>$ Classification of rules in dermatology data sets for medicine [21]

$>$ Integrating design stages for engineering using GA [22]

$>$ Multilingual question classification through GFS [23]

$>$ University admission process through evolutionary computing [24]

$>$ Genetic mining for topic based on concept distribution [25]

$>$ Intelligent web miner with Neural-Genetic-Fuzzy approach [26]

$>$ Extraction of fuzzy classification rules with genetic expression programming [27]

$>$ Integrated approach for intrusion detection system using GA [28]

\section{CaSe Study: MI with Genetic Fuzzy Methodology}

All stated efforts have not yet included evolving knowledge-base approach through genetic fuzzy system to identify specific types of intelligence. Hence, we propose the detailed design including experiments of a system to satisfy the need of decision support using GFS to achieve efficient and powerful classification of human capabilities. The architecture of proposed system is integrated through left component and right component as shown in Fig.1.

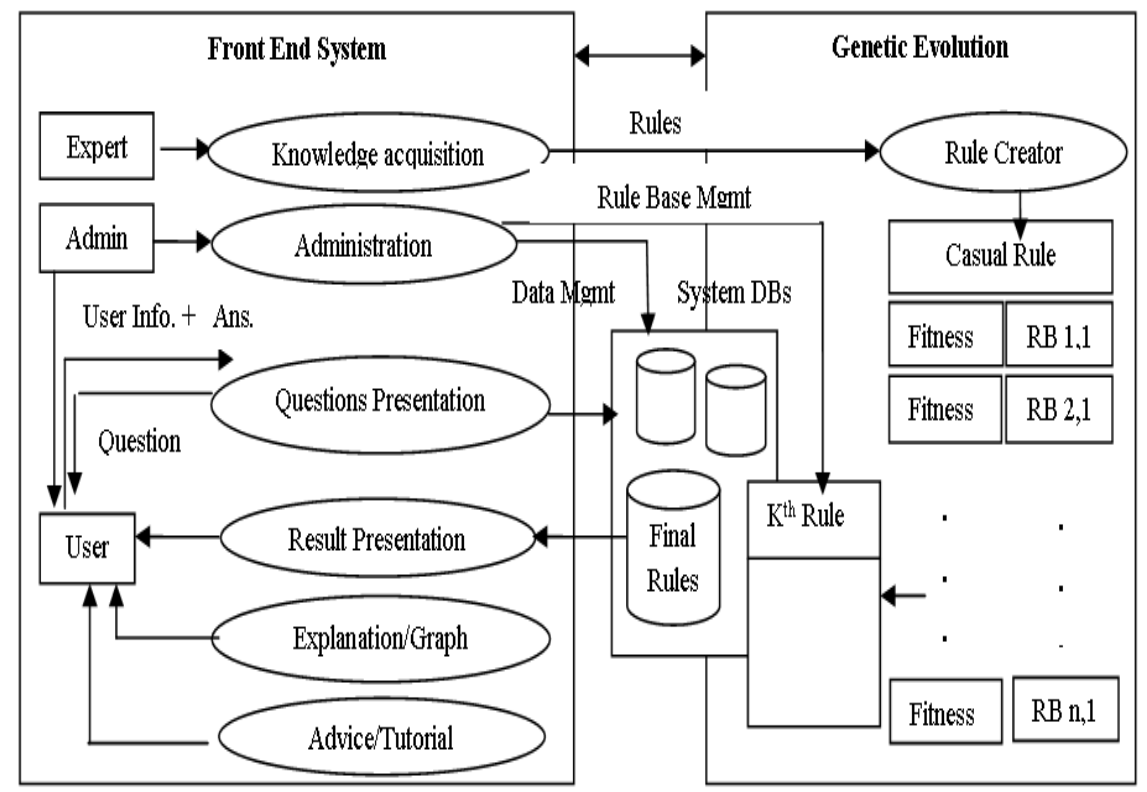

Fig. 1. Architecture of system 
International Journal on Soft Computing ( IJSC ), Vol.2, No.1, February 2011

\subsection{Detailed Methodology}

Domain knowledge can be created with set of rules which can be collected, analyzed, and finalized during interviews with experts or from multiple references as well as from example sets using theory of MI. Later, this domain knowledge is inserted and modified by human expert. Different sets of interactive questionnaires for different user categories are created/ collected by human/domain experts. Different users with their access rights will be created according to their categories; for example, higher secondary education students, college students, and professionals. According to user's category, questionnaires will be presented. User selects answer from given list of multiple choices. These answers will be stored in the database and result is shown to the users. Once score is shown to users, system provides decision using evolved rules to select appropriate class such as technical or management. The procedure of rule evolution is transparent to the users and executes in background. The users are advised to improve their intelligence by the system. In order to reinforce the intelligence; different tutorials will be suggested and presented.

\subsection{Genetic Evolution Process}

Rule base in application architecture can be generated by predefining membership functions either by a human expert or by some other processes automatically. For, proposed system, RB becomes fixed during the process. Initially, rules are suggested by human expert using different types of intelligence for efficient categorization of skills of users. Knowledge engineer facilitates rules within the rule bases in encoded fashion. Fitness of each rule is measured with fitness function. It is obvious that higher the fitness, the rule is considered as stronger. An individual is evaluated through fitness function. Application specific fitness function has been designed which calculates strength of population selected as a parent for next generation. The proposed Evolving procedure is as follows:

\section{Generate an initial population of encoded rules.}

Initial population can be generated using random number that can be assumed values from 0 to ki where ki is the number of fuzzy sets utilized to represent the attribute ai. Here, the code is obtained by concatenating rules using AND operator. The rule code is fixed and consequently location on the chromosome indicates the start and end of a particular rule code. The overall number of fuzzy sets in the DB is L[15]:

$\mathrm{L}=\mathrm{La}+\mathrm{Lc} ;$ where

$\mathrm{La}=\sum \mathrm{Ni}$, where $\mathrm{i}=1$ to $\mathrm{n}$

$\mathrm{Lc}=\sum \mathrm{Mj}$, where $\mathrm{j}=1$ to $\mathrm{m}$

Here, $n$ and $m$ are the number of input and output variables. Ni represents the number of linguistic terms associated to input variable $\mathrm{Xi}$ and $\mathrm{Mj}$ the number of linguistic terms associated to output variable $\mathrm{Cj}$. The general structure of a rule with AND operator is:

If $\mathrm{X} 1$ is $\mathrm{Y} 1 \mathrm{AND} \mathrm{X} 2$ is $\mathrm{Y} 2$ then $\mathrm{Z} 1$ is $\mathrm{C} 1$

Where $\mathrm{X} 1, \mathrm{X} 2$ are input variables, $\mathrm{Y} 1, \mathrm{Y} 2$ is Linguistic Value; $\mathrm{Z} 1$ is output variable and $\mathrm{C} 1$ is value.

Binary encoding scheme has been used to encode rule condition and prediction parts. The proposed encoding scheme is a novel approach as it deals with every label associated with the rule. A chromosome is divided into $\mathrm{n}$ genes in which each gene corresponds to a full rule. There can be many conditions in antecedent part of a rule. 
International Journal on Soft Computing ( IJSC ), Vol.2, No.1, February 2011

Table 1. Binary Representation of a Rule

\begin{tabular}{|l|c|c|c|l|c|}
\hline $\begin{array}{c}\text { Value of X1,X2 } \\
\text { (Conditional } \\
\text { Variables) }\end{array}$ & Encoding & $\begin{array}{c}\text { Value of A1,A2 } \\
\text { (Linguistic } \\
\text { Variables) }\end{array}$ & Encoding & $\begin{array}{c}\text { Consequent } \\
\text { Variables } \\
\text { (Y) }\end{array}$ & $\begin{array}{c}\text { Encoding } \\
\text { (C1) }\end{array}$ \\
\hline X1=SLogical & 1110 & High & 1001 & Y=Class & 0110 \\
\hline X2=SVerbal & 0111 & Medium & 1000 & Technical & 11 \\
\hline & & Low & 0001 & Mgmt & 01 \\
\hline
\end{tabular}

For implementing fuzzy rules, proposed system uses fuzzy mamdani membership functions used in Term set1: \{High, Medium, Low $\}$ while term set 2 consist of output label set \{Technical, Mgmt $\}$ for output variables. Different combinations from Table 3 will be utilized for chromosome representation scheme.

\section{Evaluate fitness of these rules and store into the rule profile}

Ideally, quality of rule depends on following criteria; such as high predictive accuracy, comprehensibility, and interestingness. The proposed encoding scheme focuses on predictive accuracy which is discussed as follows: Let rule be in the form: IF A then C, where A is Antecedent and $\mathrm{C}$ is consequent. The predictive performance of the rule can be summarized by $2 * 2$ matrix which is known as confusion matrix shown in Table 2 . The labels in each quadrant of the matrix have following meaning [30]:

Table 2.Confusion Matrix

1. $\mathrm{TP}=$ True

\begin{tabular}{|l|l|l|}
\hline & Actual Positive & Actual Negative \\
\hline Positive Prediction & True Positive & False Positive \\
\hline Negative Prediction & False Negative & True Negative \\
\hline & Total Positive & Total Negative \\
\hline
\end{tabular}

Positive $=$ Number of examples satisfying $\mathrm{A}$ and $\mathrm{C}$

2. $\mathrm{FP}=$ False Negative=Number of examples satisfying $\mathrm{A}$ but not $\mathrm{C}$

3. $\mathrm{FN}=$ False Negative $=$ Number of example not satisfying A but satisfying $\mathrm{C}$

4. $\mathrm{TN}=$ True Negatives=Number of examples not satisfying A nor $\mathrm{C}$

Hence, $\mathrm{CF}($ Precision $)=\mathrm{TP} /(\mathrm{TP}+\mathrm{FP})$

Predictive accuracy is measured by (3) by finding proportion of examples having predicted class $\mathrm{C}$ that is actually covered by rule antecedent. The rule completeness (true positive rate) can be measured by following equation.

$\mathrm{Comp}=\mathrm{TP} /(\mathrm{TP}+\mathrm{FN})$

By combining (3) and (4) we get; 
Fitness $=\mathrm{CF}^{*} \mathrm{Comp}$

\section{Determine the fitness accepted for the application.}

The individual rules are tested for the fitness and result is stored into appropriate rule profile. One may start with some default general rules within an initial population. For each rule, degree of fitness is calculated according to the above mentioned fitness function. According to a defined termination criterion, new offspring is generated. Using this methodology, a stronger rule can evolve with every new generation.

\section{Identify and discard the weak rules according to rule matching criteria.}

As it is a binary encoding scheme, every time result of last operation is calculated using following criteria:

Decimal Value of chromosome from initial population is calculated and stored in DB. After every iterative cycle, value of new chromosome will be is calculated and compared along with its label stored in DB.

\section{Apply crossover and mutation operators on rules}

According to the theory of GA, a crossover operator selects substrings of genes of the same length from parent individuals which are known as off-springs from the same point, replaces them and generates a new individual. This point can be selected randomly [29]. For designing chromosome, we have used binary encoding style as shown in Table 1.Different rules from Table 3 can be represented in form of chromosomes labeled as individuals. Here, single point crossover operator has been implemented.

Rule 1: If SLogical is High and SVerbal is Low then class is Technical

Individual 1(I1): 111010010111000111111

Rule 2: If SLogical is Medium and SVerbal is High then class is Mgmt

Individual 2(I2): 111010000111100111101

$\begin{array}{llllllllllllllllllllll}\text { I } 1 & 1 & 1 & 1 & 0 & 1 & 0 & 0 & 1 & 0 & 1 & 1 & 1 & 0 & 0 & 0 & 1 & 1 & 1 & 1 & 1 & 1 \\ \text { I } 2 & 1 & 1 & 1 & 0 & 1 & 0 & 0 & 0 & 0 & 1 & 1 & 1 & 1 & 0 & 0 & 1 & 1 & 1 & 1 & 0 & 1\end{array}$

Fig.2 First Generation Individuals

Fig. 2 shows rules representation in form of chromosome. One point cross over operator is applied on individuals. This operation interchanges the bit string from cut off position at randomly selected point from rule conditional part. As an outcome, we get Fig. 3 which represents new individuals from next generation.

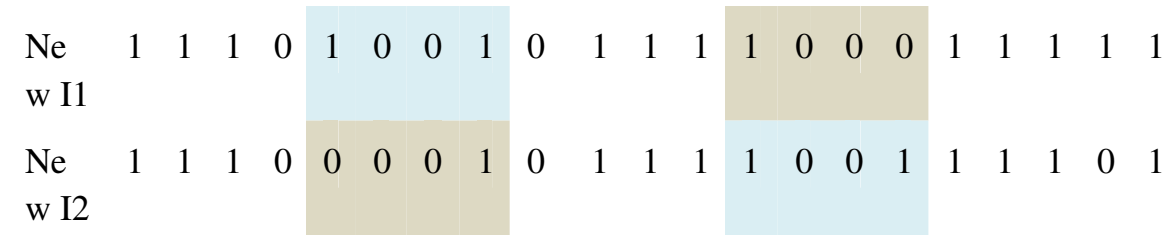

Fig. 3. Next Generation of Individual as a Result of Cross Over

Finally, as a result of decoding process, we get following new rules in form of off-springs from result of cross over operation.

New rule 1: If SLogical is High and SVerbal is Medium Then Class is Technical

New rule 2: If SLogical is Low and SVerbal is High Then Class is Mgmt 
After applying rule matching process, the output of crossover operator has generated two new rules which are also available in rule sets as shown in Table 3 [31].

Fig. 4 shows result of mutation operator randomly selects the locus on new chromosome which does inversion on bits from 0 to 1 and 1 to 0 at location with a set probability Pm. Mutation causes the individual genetic representation to be changed according to some probabilistic rule.

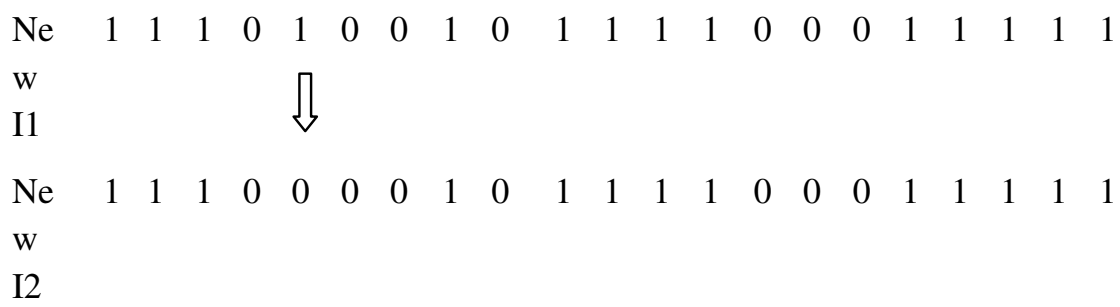

Fig. 4 Result of mutation operator

\section{Go to step (ii) and repeat the procedure till required fit rules are achieved.}

Hence, we can determine that using proposed scheme new feasible rules can be evolved in upcoming generations. Here, Table 3 shows rule set is designed by human expert to identify technical and managerial skills of human being.

Table 3. Rule Set Identifying Class

\begin{tabular}{|c|l|}
\hline 1 & If SLogical is High and SVerbal is Low then class is Technical \\
\hline 2 & If SLogical is High and SVerbal is High then Class is (Technical OR Mgmt) \\
\hline 3 & If SLogical is High and SVerbal is Medium then Class is Technical \\
\hline 4 & If SLogical is Medium and SVerbal is Medium then Class is (Technical OR Mgmt ) \\
\hline 5 & If SLogical is Medium and SVerbal is Low then Class is Technical \\
\hline 6 & If SLogical is Medium and SVerbal is High then Class is Mgmt \\
\hline 7 & If SLogical is Low and SVerbal is High then Class is Mgmt \\
\hline 8 & If SLogical is Low and SVerbal is Medium then Class is Mgmt \\
\hline 9 & If SLogical is Low and SVerbal is Low then Class is Rejected \\
\hline
\end{tabular}

\section{Experiments \& Results}

For good performance of the system, the design of fuzzy membership function is very important. Here, Mamdani FIS is used for implementation of fuzzy inference mechanism. Three different gaussian membership functions (Low, Medium, and High) have been used to represent degree of truth of two input (conditional) variables: SLogical and SVerbal. For output variables, triangular membership functions have been used. "AND" method is used as a part of aggregation and "Centroid" method is used for de-fuzzification. The rule base consists of nine rules. Fig. 5 shows the sample output of fuzzy-mamdani membership function plotting with MATLAB 7.0. Over many generations, natural population evolves according to principle of evolutionary computation. By continuing the method of automatic evolution, self tuning of membership function became possible. 


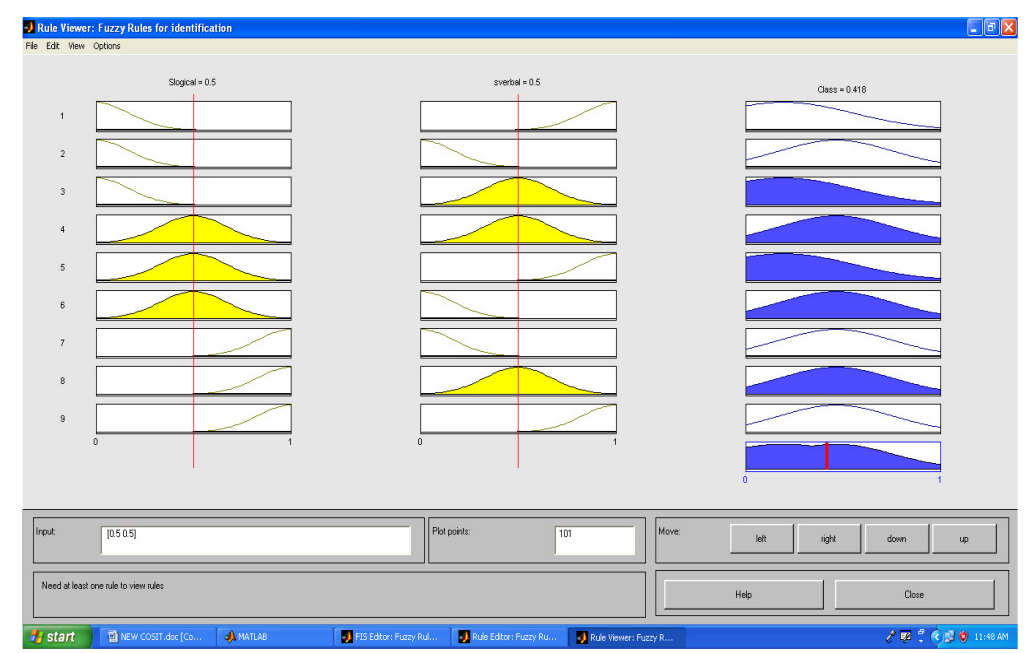

Fig.5. Evolved Rules

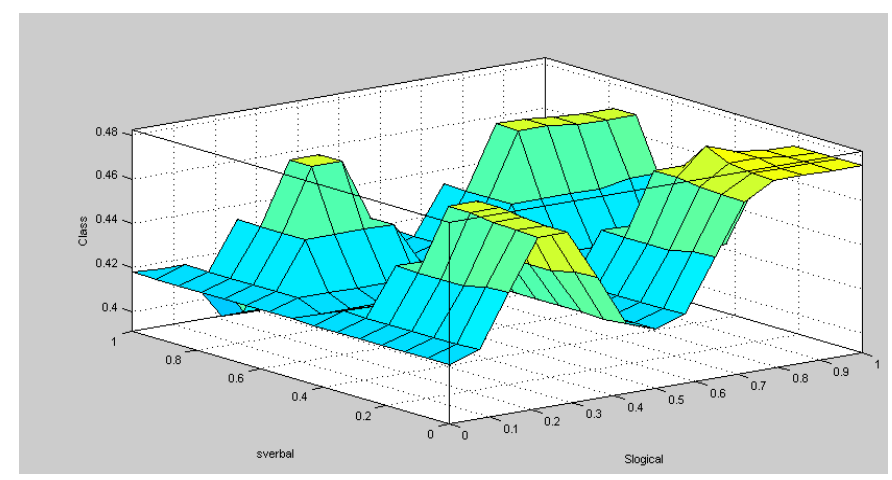

Fig. 6 Surface viewer of MI rule set

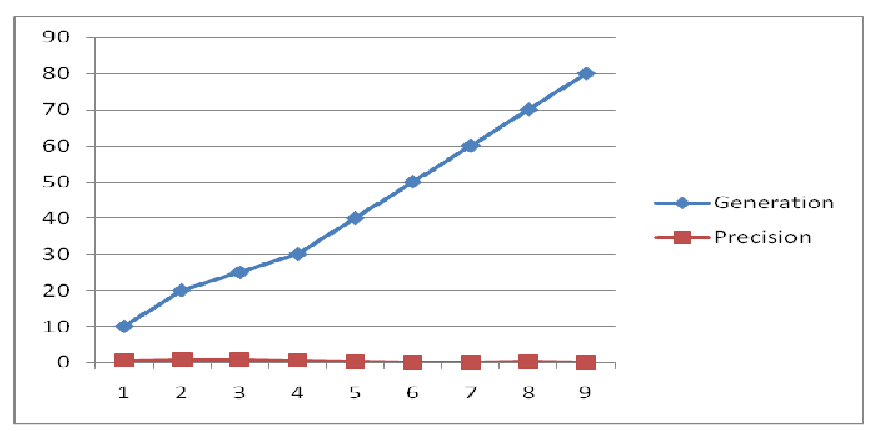

Fig.7 Generation Vs Precision

Fig. 7 shows implementation of GA with following parameters:

No of chromosomes in populations: 16

Crossover probability:0.6

Chromosome mutation probability:0.05

Random selection probability : 0.1

Total No of generations: 80

We achieved precision $=0.8$ and completeness $=0.99$, through implementation of GA with proposed methodology. Fig. 8 shows, accuracy rate for every 10 generations. 


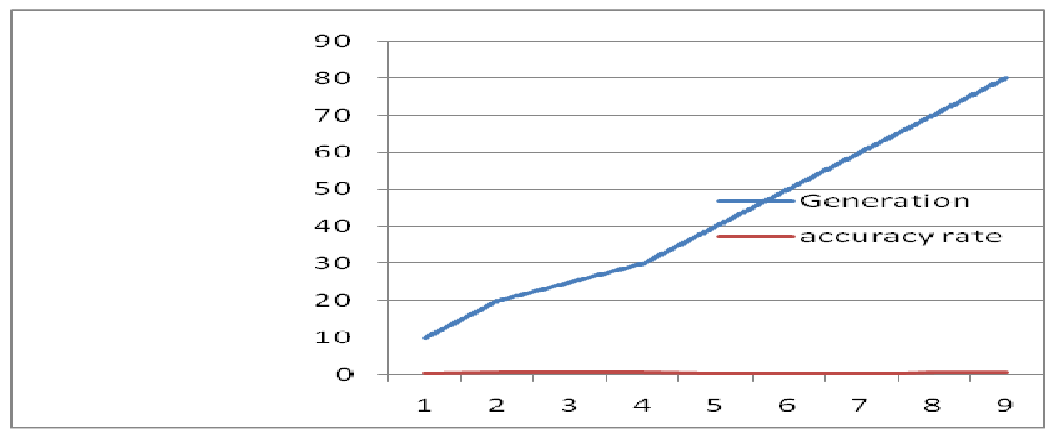

Fig.8 Generation Vs Accuracy rate

Result of questionnaires is calculated with the help of total score for every student. Three different criteria: High, Low, Mid are determined to analyze the results. Fig. 9 shows the total number of students acquiring specific type intelligence i.e. Logical, Verbal as an implementation of proposed system.

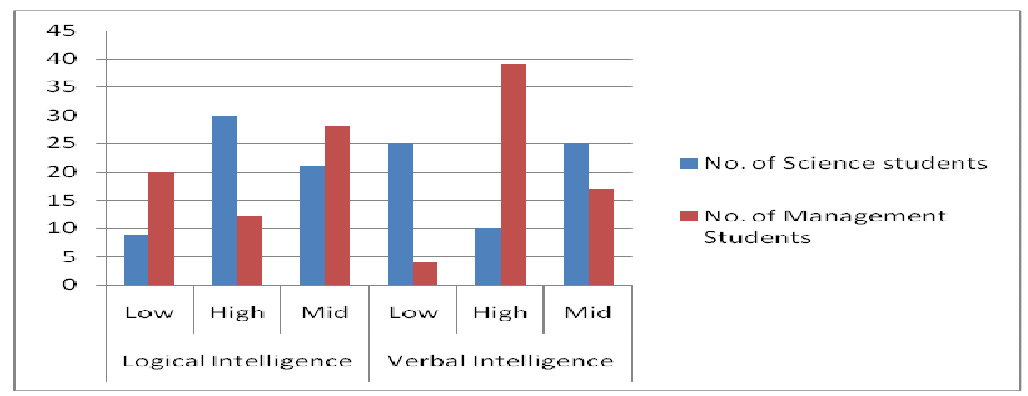

Fig.9. Intelligence Type Vs No. of Students

\section{Conclusion and Future Work}

The presented application is an intelligent system design to identify students' different skills in education domain. It offers many advantages such as handling imprecision. The framework of system is generalized which can be mapped into different types of applications which reduces efforts for creation and documentation of knowledge. The architecture of evolving rule based model using genetic-fuzzy approach can also be applied to various domains like advisory systems, decision support systems, data mining systems, and control and monitoring systems, etc. The same approach can be used to provide training for teachers, planning for resources and many more.

Further, new hybrid operator can be identified using suggested encoding schemes. The system can also be extended to different areas where analysis of human intelligence is required. New inventions in Multiple Intelligence can also be integrated with designed rule sets. The proposed system inaugurates a novel platform for hybridization of front end system with automatic evolution from intelligent system design. A generic commercial product with an interactive editor in the domain of multiple intelligence identification can be available. This increases the scope of the system and meets the requirements of increased number of non-computer professionals in various fields.

\section{References}

1. Carter, P.: The Complete Book of Intelligence Tests. John Wiley,0-470-01773-2 (2005)

2. Gardner, H.: Multiple Intelligences After Twenty Years. American Educational Research Association, Chicago, Illinois (2000) 
International Journal on Soft Computing ( IJSC ), Vol.2, No.1, February 2011

3. Motah, M.: The Influence of Intelligence and Personality on the Use of Soft Skill in Research Projects among Final year University Students: A Case Study. In: Proceedings of Informing Science \& IT Education Conference (InSTE), Mauritius (2008)

4. Intan, S., Faris, Z., Norzaidi, M., Normah, O.: Multiple Intelligences Educational Courseware: Learning Tool for Malaysia Smart School. In: Proceedings of EABR \& TLC Conferences Proceedings, Germany (2008)

5. Sternberg, R.J.: Abilities are forms of Developing Expertise. American Educational Research Association, Vol. 27, No. 3, pp.11-20 (1998)

6. Harward School of Education, http://www.pz.harvard.edu/Research/SUMIT.htm

7. Dara, P.A.: Applying Multi-Intelligent Adaptive Hypermedia Online Learning, E-Learn 2002.In: Proceedings of Conference at Association for the Advancement of Computing in Education (AACE), Canada (2002)

8. Kaur, G., Chhikara, S.: Assessment of Multiple Intelligence among Young Adolescents (12-14 Years). J. Hum. Ecol., 23(1): 7-11 (2008)

9. National Center for the Study of Adult Learning and Literacy, http://www.pz.harvard.edu/Research/AMI.htm

10. Kelly, D.: On the Dynamic Multiple Intelligence Information Personalization the Learning Environment, Ph.D. Thesis, University of Dublin (2005)

11. Rasmani, K., Shen, Q.: Data-Driven Fuzzy Rule Generation and its Application for Student Academic Performance Evaluation, Journal of Applied Intelligence 25(3):305-319 (2006)

12. http://stutzfamily.com/mrstutz/APPsych/thoughtandlanguage/lecturenotesintelligence.html

13. Alvaro, C., Norian, M., Aledir, S.: Application of the Theory of Multiple Intelligences to Digital Systems Teaching. In: Proceedings of 39th ASEE/IEEE Frontiers in Education Conference, San Antonio (2009)

14. Herrera, F.: Ten Lectures on Genetic Fuzzy Systems, Technical report ,SCCH-TR-0021, Spain (1997)

15. Cordon, O., Herrera, F., Hoffmann, F., Magdalena, L.: Genetic Fuzzy Systems, Evolutionary Tuning and Learning of Fuzzy Knowledge Bases, Advances in Fuzzy system-Applications and Theory, Vol. 19, pp.89-93,97,179-183, World Scientific, USA (2001)

16. Didelis, M.V., Lopes, H.S., Freitas, A.A..: Discovering Comprehensible Classification Rules with a Genetic Algorithm, Brazil (2000)

17. Sajja, P.S.: An Evolutionary Fuzzy Rule Based System for Knowledge Based Diagnosis. JHCR J. Hybrid Computing Research 2(1) (2009)

18. Cheung, W., Kaymak, U.: A fuzzy Logic Based Trading System, Technical Report, Erasmus Institute ,The Netherlands (2007)

19. Sonja, P., Abhraham, A., Ken, C.: EvoPol- A framework for optimizing social regulation policies. Kybernetes, 35 (6):814-826 (2003)

20. Wang, C., Hong, T., Tseng, S.: A Genetics -Based Approach to Knowledge Integration and Refinement. Journal of Information Science and Engineering 17,85-94 (2001)

21. Herrera, F.: Genetic Fuzzy Systems: Status, Critical Considerations and Future Direction, Journal of Computational Intelligence Research,0973-1873, Vol.1, 59-67 (2005)

22. Lee, M., Takagi, H.: Integrating Design Stages of Fuzzy Systems using Genetic Algorithms. In: Proceedings of 2nd International Conference on Fuzzy systems. vol.1,pp. 612-617.IEEE Press, CA (1997)

23. Day, M., Ong, C., Hsu, W.: Question Classification in English-Chinese Cross-Language Question Answering: An Integrated Genetic Algorithm and Machine Learning Approach, Technical Report, Institute of Information Science \& Academia Sinica \& Department of Information Management, Taiwan (2007)

24. Serag-Eldin, G., Souafi-Bensafi., S., Lee, J., Chan, W., Nikravesh, M.: Web Intelligence: Web-Based BISC Decision Support System (WBISC-DSS).BISC Program, CA (2002)

25. Khaliessizadeh, S.M., Zaefarian, R., Nasseri, S.H., Ardil, E.: Genetic Mining: Using Genetic Algorithm for Topic based on concept Distribution. In: Proceedings of World Academy of Science, Engineering and Technology, Vol.13,1307-8884 (2006)

26. Abhraham, A., Wang, X.: i- Miner: A Web Usage Mining Framework Using Neuro-Genetic-Fuzzy Approach. Department of Computer Science(USA), School of Business System, Australia (2003)

27. Marghhny M., EI, S.: Extracting Fuzzy Classification rules with gene expression Programming. In: Proceedings of AIML 05 Conference, CICC, Egypt (2005)

28. Skevajabu, K, Rebgan, S.: Integrated Intrusion detection system using soft computing, International J. Network Security 10, 87-92 (2010) 
29. Akerakar, R., Sajja, P.S., Knowledge-Based Systems, Jones and Bartlett, Massachusetts (2010)

30. Freitas, A.: A Survey of Evolutionary Algorithms for Data Mining and Knowledge Discovery, 31-36, AAAI Press, Brazil (2003)

31. Kunjal, M., Sajja, P.S.: A design of encoding strategy and fitness function for Genetic-Fuzzy system for classification of students' skills. In: proceedings of 1st International Conference on Signals, Systems and Automation(ICSSA 2009), Vallabh Vidyanagar, India (2009)

\section{Biography}

\section{Kunjal Mankad}

Kunjal Mankad (1977) joined as a Lecturer at MCA Department of Institute of Science \& Technology for Advanced Studies \& Research; Vallabh Vidyanagar in 2002. She is presently working as assistant professor. She received her MCA from department of computer science, Saurashtra University, Rajkot. She has been working as a Ph.D. student for research work under doctoral supervision of Dr. Priti Sajja. Her research area is genetic fuzzy system. She has published/presented several papers in International and National Journals and Conferences. One of her research paper has received 2nd best paper award. She is a member of board of studies of Sardar Patel University, Vallabh Vidyanagar. She is serving as editorial board member of international journal in computer science and technology.

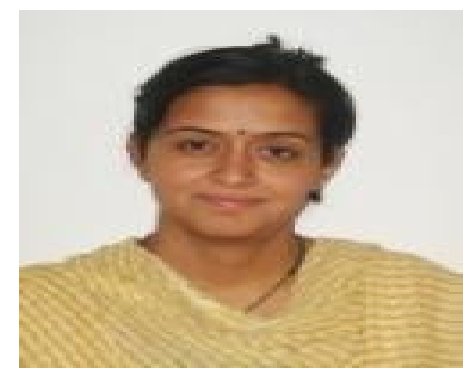

\section{Dr. Priti Srinivas Sajja}

Dr Priti Srinivas Sajja (b.1970) joined the faculty of the Department of Computer Science, Sardar Patel University, India in 1994 and presently working as an Associate Professor. She received her M.S. (1993) and Ph.D (2000) in Computer Science from the Sardar Patel University. Her research interests include knowledge-based systems, soft computing, multi-agent systems, and software engineering. She has 81 publications in books, book chapters, journals, and in the proceedings of national and international conferences. Three of her publications have won best research paper awards. She is co-author of 'Knowledge-Based Systems' published by Jones \& Bartlett Publishers, USA. She is supervising work of seven doctoral research students. She is Principal Investigator of a major research project funded by UGC, India. She is serving as a member in editorial board of many international science journals and served as program committee member for various international conferences.

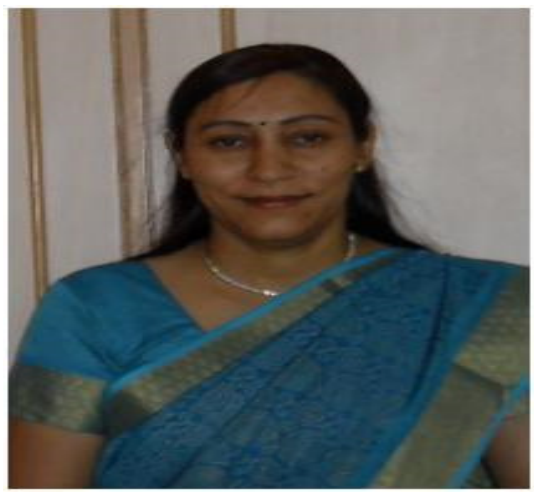




\section{Dr. Rajendra Akerkar}

Rajendra Akerkar is a professor of computer science and holds academic assignments in India and abroad. As founder, he is instrumental in ensuring that the Technomathematics Research Foundation (TMRF) lends a platform for research in India. Under his leadership, TMRF has become one of the well-known organizations amongst the research community worldwide. He holds position as a professor/senior researcher at the Western Norway Research Institute (Vestlandsforsking), Norway. His research experience includes over 18 years in the Academia spanning different universities in Asia, Europe and North America. His current research agenda basically concentrates on learning and language --- how each works in the human and how they can be replicated in a machine.

He received DAAD fellowship in 1990 and also awarded prestigious BOYSCASTS Young Scientist award of Department of Science \& Technology, Government of India, in 1997. He is an Editor-in-Chief of International Journal of Computer Science \& Applications and Associate Editor of International Journal of Metadata, Semantics and Ontologies (IJMSO). He is involved as the scientific committee member of many international conferences and also serves the editorial board of international journals in computer science. He has authored 11 books, more than 90 research papers and edited 4 volumes of international conferences in Artificial Intelligence \& Applications of Computer Algebra. He is actively involved in many industrial research and development projects for more than 14 years.

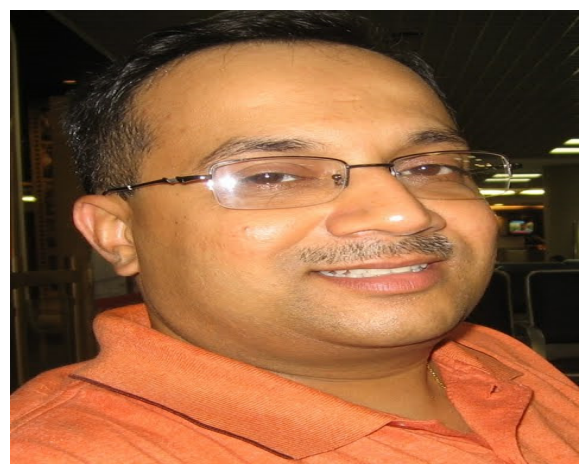

